\title{
Role of nanomedicines in cell-based therapeutics
}

\author{
Z haoyang Ye' \& \\ Ram I Mahato ${ }^{2 \dagger}$ \\ ${ }^{\dagger}$ Author for correspondence \\ ${ }^{1}$ T he Johns H opkins \\ U niversity, D epartment of \\ Biomedical Enginering, \\ 3400 N. Charles Street/Clark \\ $\mathrm{H}$ all 102, Baltimore, \\ M D 21218, USA \\ Tel.: +1 443572 9007; \\ Fax: +1 410516 8152; \\ Email: zye5@jhu.edu \\ $2 U$ niversity of Tennessee \\ $\mathrm{H}$ ealth Science Center, \\ $D$ epartment of \\ Pharmaceutical Sciences, \\ 19 S. M anassas, RM 224, \\ M emphis, T N 38103-3308, \\ USA \\ Tel.: +1 901448 2099; \\ Fax: +1 901448 6929; \\ Email: rmahato@utmem. \\ edu
}

\section{'Cell-based therapeutic shasgreat potential to treat both genetic and acquired diseases.'}

Cell-based therapeutics is one of the most rapidly growing fields in translational medicine. It stands at the intersection of a variety of rapidly developing disciplines, including biomaterials, transplantation, tissue engineering and regeneration and stem cell biology. Cell-based therapeutics has great potential to treat both genetic and acquired diseases [1]. Blood transfusions and bone marrow transplantation are two typical examples of cellbased therapeutics, however, recent advances in cell and molecular biology have expanded the potential applications of this approach. Genetically engineered cells can be used as therapeutics. Cells are being used increasingly as drug-delivery vehicles. Currently, stem cells are becoming rising stars in cell-based therapeutics for tissue engineering and cell-replacement therapy because of their pluripotency and self-renewal capability. There fore, molecular-level or nanoscale control over performance of cells is envisioned for modern cell-based therapeutics.

$\mathrm{N}$ anomedicine is an offshoot of nanotechnology and refers to highly specific medical intervention at the molecular scale for curing diseases or repairing tissues $[2,3]$. As a new interdisciplinary field, it has drawn great interest in both academia and industries. There are al ready several commercialized products based on nanomedicine, including drug delivery, diagnostics, imaging, biomaterials and implants [2]. $\mathrm{N}$ anomedicines will certainly have an important role in cell-based therapeutics and thus have already been used successfully for improved immunoprotection of transplanted cells and tissues and for tissue regeneration, as discussed later. The application of nanotechnologies to cell-based therapeutics must expand the area of nanomedicine.

Nanomaterials for immunoisolation of cells \& tissues

Cell/tissue transplantation is a typical example of cell-based therapy. Immune attack by the host immune system is one of the major challenges in cell transplantation. Immunoisolation of transplanted islets through semi-permeable membranes is an attractive strategy to block the infiltration of immune cells, such as leukocytes and macrophages, to the transplantation site [4]. $\mathrm{N}$ anosized structures and nanoporous materials can be used for immunoisolation of cells and pancreatic islets. For example, cells could be placed into chambers fabricated with bulk micromachining within single crystalline silicon wafers [5]. The chambers interface with the surrounding biological environment through polycrystalline silicon filter membranes micromachined to present a high density of uniform nanopores as small as $20 \mathrm{~nm}$ in diameter. Encapsulated rat pancreatic cells receive nutrients and remain healthy for weeks in vitro but remain protected from the immune system, which would normally attack and reject foreign cells.

However, to protect islets from immunemediated destruction, camouflaging the surface of islets at the molecular level shows promising results for immunoisolation and immunoprotection. Two major approaches have been tried to prevent immunogenic reactions of the cellular surface. $O$ ne is microencapsulation of the cells and the other is surface modification of the cells.

Most immunoisolation strategies use microcapsules consisting of cells or cell clusters entrapped within a spherical semi-permeable membrane. Poly(vinyl alcohol) (PVA), poly(D,Llactide-co-glycolide) (PLGA), dimethylaminoethyl methacrylate methyl methacrylate copolymer, alginate with or without poly(L-lysine) (PLL) and agarose have been used for the encapsulation of islets. Microencapsulation of islets by the PLL-alginate polymer complex is the most succesful example of this technology. The semi-permeable membrane permits nutrient flow and oxygen transport but prevents immunogenic reactions. H owever, this technology has several limitations, including reduced life span of the cells owing to polymer biodegradation, permeability of the capsule, fragility and limited surface area.

The idea of molecular camouflage is through the immobilization of polymer chains (normally polyethylene glycol [PEG]) to the cell or tissue surface, creating a molecular barrier of PEG that 
prevents molecular recognition between cellsurface receptors and soluble ligands. G enerally, this has been accomplished through covalent coupling of PEG to amines of cell-surface proteins or carbohydrates or by direct insertion of PEG - lipid conjugates into the cell membrane [6]. PEG is a hydrated, flexible polymer chain comprising highly mobile repeating units, which enable the polymer chain to act as a steric barrier on the cell surface [7]. PEGylation of islets [8] helps maintain normoglycemia for more than 100 days without any immunosuppressants, possibly owing to prevention of graft infiltration by host immune cells. H owever, the efficacy and stability of PEGylation for immunoisolation might be limited owing to the lack of a defined pore structure and dependence on a steric exclusion effect to provide an immunoprotective barrier, as well as the dynamic turn-over of the cell-surface components [9].

\footnotetext{
'Nanomedic ine is an offshoot of na notechnology and refers to highly specific medical intervention at the molecular scale for curing diseases or repa iring tissues. As a new interd isc ip linary field, it has d rawn great interest in both academia and industries.'
}

Alternatively, ultra-thin film build-up through electrostatic layer-by-layer assembly is also particularly attractive for cell-coating applications [10]. Through this process, the negatively charged cell membrane can be encased within a cationic polymeric shell; oppositely charged polymers can be adsorbed subsequently to achieve a desired thickness and surface composition. The thickness of polyelectrolyte multilayers (usually $5-50 \mathrm{~nm}$ ), composition and number of layers will affect capsule permeability. Encapsulation of islets with three layers composed of phospholipid-PEG, sodium alginate and PLL has been reported, which did not impair the insulin-release function in response to glucose stimulation [11]. Succesfful encapsulation of stem cells with layers of PLL and hyaluronic acid has also been reported [12]. Although the immunoisolation capacity of these coating materials has not yet been demonstrated, deposition of ultra-thin, multilayer films directly on the surface of cells and tissues is a promising approach for minimizing void volume while generating a true permselective membrane. Following this trend, the successful clinical use of immunoisolation will not be far way.

\section{Biomimetic materials for}

tissue engineering

Tissue engineering, al so called regenerative medicine, is another example of cell-based therapy. Stem and progenitor cells have the potential to replace cells that are damaged or diseased and to restore vital functions. Integration of basic and translational research supports the development of new cellular therapies. Although stem cells are the key players in current tissue engineering, issues pertaining to the maintenance and expansion of the undifferentiated population in vitro and to control directed differentiation in vitro as well as in vivo are still not well addressed. N anotechnology provides a promising opportunity for tissue engineering. Extracellular matrix (ECM) is the natural scaffold for cells, tissue and organ growth. $N$ ative ECM does far more than just provide a physical support for cells. It al so provides a substrate with specific ligands for cell adhesion and migration and regulates cellular proliferation and function by providing various growth factors. Therefore, it is reasonable to expect that an ECM mimicking tissue-engineered scaffold will have a similar role to promote tissue regeneration in vitro as native ECM does in vivo. Any scaffold material must be able to interact with cells in three dimensions and facilitate this communication. However, to mimic the structural features of natural ECM might be the easiest step to start with for developing scaffolds in tissue engineering.

A well-known feature of native ECM is the nanoscaled dimensions of their physical structure. The structural ECM proteins, such as colla gen fibers and elastin fibers, range in diameter from 50 to $500 \mathrm{~nm}$. 0 ther components in ECM, such as adhesive proteins, including fibronectin and laminin, which present specific binding sites for cells, also exist as nanofibers. In addition, the fibers, pores, ridges and grooves on the basement membrane are all nanoscaled, from several to more than $100 \mathrm{~nm}$ [13]. The topology of ECM has an important role in cell behavior [14,15]. Therefore, one requirement for tissue engineering scaffolds is the mimicry of nanoscale structure of the ECM . H owever, there is still a design challenge to fabricate biomaterials that mimic ECM 's 3D structures with defined shapes and complex porous architecture.

O ne typical example is nanofibers. $\mathrm{N}$ anofibers fabricated by self-assembly, phase separation or electrospinning are promising candidates to mimic natural ECM as scaffolds for tissue engineering [16]. Both self-assembly and phase-separation techniques have been used successfully to 
fabricate nanofibers for tissue engineering. However, in comparison, currently, electrospinning is widely used by researchers because of the simplicity, diversity and control over scaffold geometries and mechanical characteristics, the easy scaling-up property. Electrospinning is applicable to many different polymers, even though the diameters of electrospun fibers generally reside on the upper limits of the natural ECM 's 50-500 nm range [16]. Both synthetic polymers, such as poly(glycolic acid) (PGA), poly(lactic acid) (PLA), polydioxanone, polycaprolactone $(P C L)$ and their blends or copolymers, and natural polymers of ECM components, such as elastin and collagens, have been exploited [16]. Further incorporation of glycosaminoglycans (GAG) into an ECM analog during electrospinning could potentially be an important aspect in truly mimicking the native ECM . GAG s serve a variety of functions, including linking collagen structures and binding growth factors. The specific GAGs of physiological and tissue engineering scaffolds are hyaluronic acid, dermatan sulfate, chondroitin sulfate (most abundant GAG in tissues), heparin, heparan sulfate and keratan sulfate. $M$ any different nanofibers support the adhesion, proliferation and differentiation of adult stem cells [17]. A 3D construct made of a multilayer organization of nanofibers supported human mesenchymal stem cell proliferation [18]. After in vivo implanting, the multilayer scaffold was integrated fully with the surrounding tissues, having a cell density comparable with the surrounding tissue; the engrafted construct supported neovascularization, which is imperative for the survival of any implant and tissue [18]. Nanofiber scaffolds have found themselves promising applications in engineering many different tissues, such as wound dressing, skin, blood vessel, nerve, bone and cartilage [19].

Further modifications of nan ofibers have produced more functional scaffolds for tissue engineering. Functionalizing nanofibers can be achieved by several methods [19]: to make composite nanofibers by direct electrospinning, surface modification of nanofibrous scaffolds after electrospinning or using a newly developed electrospinning technology, coaxial electrospinning technique. Coaxial electrospinning can produce a core-shell structured composite fiber to fulfill different application purposes, especially for controlled growth factor or drug release in tissue engineering. Coaxial electrospinning of aligned
PCL nanofibers encapsulated with bovine serum albumin and platelet-derived growth factor-BB for controlled drug release was achieved [20]. These nanofibers can find potential use in tissue engineering. Incorporation of different chemical components into nanofibers can also be possible. For example, nanofibers of PEG-blockPCL-block copolymers with end modification of Arg-Gly-Asp (RGD) peptides helped the adhesion and survival of fibroblasts [21]. Collagen-coated nanofibers also enhance their performance for fibroblasts [22] and neural stem cells [23]. H owever, whether post-chemical modifications on nanofibers affect the structures of the nanofibers is not known yet.

'Combination of the concepts of nanomedic ine and cell-based thera peutic s will definitely boost the development of cell-based thera peutics.'

$\mathrm{N}$ anopatterning provides another technique to present nanostructural and biological clues in synthetic scaffolds, mimicking what native ECM does to cells. In brief, it is to site-specifically immobilize biomolecules onto surfaces at the nanoscaled level [24]. Patterning peptides, which signal interaction between cells and ECM, could lead to greater control over the cell-biomaterial interface. It appreciates the significance of the way that cells interact with their surrounding biological clues in more details. H owever, most of nanopatterning is carried out with substrate materials, such as silicon, $\mathrm{SiO}_{2}$ and gold [24], which are not suitable for tissue engineering. One recent study showed that nanopatterning of RGD ligands on alginate affects focal-adhesion kinase tyrosine 397 (Y397) phosphorylation, cell spreading and osteogenic differentiation, independent of RGD bulk density of M C 3T 3-E 1 preosteoblasts [25]. In detail, the distribution of islands throughout the alginate hydrogel, which meant how closely spaced the islands were, was the most significant pattern parameter. Closely spaced islands favor FAK Y 397 phosphorylation and cell spreading, whereas widely spaced islands favor differentiation.

\section{Conclusion}

Combination of the concepts of nanomedicine and cell-based therapeutics will definitely boost the development of cell-based therapeutics. C ell-based therapeutics relies heavily on the new 
materials for delivering, maintaining and providing biological clues for cells. M imicking the biological microenvironments is the straightforward way for engineering tissues from stem cells. Therefore, we can envision that synthetic materials with controlled properties at the nanoscaled level in combination with the incorporation of the molecular level of biological clues fit these niches.
Financial \& competing interests disclosure

The authors have no relevant affiliations or financial involve ment with any organization or entity with a financial interest in or financial conflict with the subject matter or materials dis cussed in the manuscript. This indudes employment, consultancies, honoraria, sock ownership or options, expert testimony, grants or patents received or pending, or royalties.

No writing assistance was utilized in the production of this manuscript.

\section{Bibliography}

1. YeZ, M ahato RI: Emerging trends in cell-based therapies. Adv. D rug D eliv. Rev. 60(2), 89-90 (2007).

2. Wagner V, D ullaart A, Bock AK, Zweck A: The emerging nanomedicine landscape. Nat. Biotechnol. 24, 1211-1217 (2006).

3. Freitas RA Jr: What is nanomedicine? Dis. M on. 51, 325-341 (2005).

4. N arang AS, M ahato RI: Biological and biomaterial approaches for improved islet transplantation. Pharmacol. Rev. 58, 194-243 (2006).

5. D esai TA, Chu WH, Tu JK, Beattie GM, $H$ ayek $A$, Ferrari $M$ : M icrofabricated immunoisolating biocapsules. Biotechnol. Bioeng. 57, 118-120 (1998).

6. Kellam B, D e Bank PA, Shakesheff KM : Chemical modification of mammalian cell surfaces. Chem. Soc. Rev. 32, 327-337 (2003).

7. Contreras JL, Xie D, M ays J et al.: A novel approach to xenotransplantation combining surface engineering and genetic modification of isolated adult porcine islets. Surgery 136, 537-547 (2004).

8. Lee DY, Park SJ, LeeS, N am JH, Byun Y: $\mathrm{H}$ ighly poly(ethylene) glycolylated islets improve long-term islet allograft survival without immunosuppressive medication. TissueEng. 13, 2133-2141 (2007).

9. Wilson JT, Chaik of EL: Challenges and emerging technologies in the immunoisolation of cells and tissues. Adv. D rug D eliv. Rev. 60(2), 124-45 (2007).

10. Leguen E, Chassepot A, D echer G, Schaaf P, Voegel JC, Jessel N : Bioactive coatings based on polyelectrolyte multilayer architectures functionalized by embedded proteins, peptides or drugs. Biomol. Eng. 24, 33-41 (2007).

11. M iura S, Teramura Y, I wata $\mathrm{H}$ : Encapsulation of islets with ultra-thin polyion complex membrane through poly(ethylene glycol)-phospholipids anchored to cell membrane. Biomaterials 27, 5828-5835 (2006).

12. Veerabadran NG, Goli PL, Stewart-Clark SS, Lvov YM , M ills D K: $\mathrm{N}$ anoencapsulation of stem cells within polyelectrolyte multilayer shells. M acromol. Biosci. 7, 877-882 (2007).

13. Flemming RG, M urphy CJ, Abrams GA, Goodman SL, N ealey PF: Effects of synthetic micro- and nano-structured surfaces on cell behavior. Biomaterials 20, 573-588 (1999).

14. M ingyu $S$, Fashui $H, C$ hao $L$ et al.: Effects of nano-anatase $\mathrm{TiO}_{2}$ on absorption, distribution of light, and photoreduction activities of chloroplast membrane of spinach. Biol. TraceElem. Res. 118, 120-130 (2007).

15. Elias KL, Price RL, Webster TJ: Enhanced functions of osteoblasts on nanometer diameter carbon fibers. Biomaterials 23, 3279-3287 (2002).

16. Barnes $C P$, Sell $S A$, Boland ED, Simpson DG, Bowlin GL: N anofiber technology: D esigning the next generation of tissue engineering scaffolds. Adv. D rug Deliv. Rev. 59, 1413-1433 (2007).

17. M a Z, Kotaki M, Inai R, Ramakrishna S: Potential of nanofiber matrix as tissue-engineering scaffolds. Tissue Eng. 11, 101-109 (2005).
18. Srouji SS, Pagan $Y L$, D 'Amato $F$ et al.: Pharmacokinetic factors contribute to the inverse relationship between luteinizing hormone and body mass index in polycystic ovarian syndrome. J. Clin. Endocrinol. M etab. 92, 1347-1352 (2007).

19. Venugopal J, Low S, Choon AT, Ramakrishna S: Interaction of cells and nanofiber scaffolds in tissue engineering. J. Biomed. M ater. Res. B Appl. Biomater. 84, 34-48 (2008).

20. Liao IC, Chew SY, Leong KW : Aligned core-shell nanofibers delivering bioactive proteins. Nanomed. 1, 465-471 (2006).

21. Grafahrend D, Lleixa Calvet J, Salber J, D alton PD, M oeller M, Klee D: Biofunctionalized poly(ethylene glycol)block-poly(epsilon-caprolactone) nanofibers for tissue engineering. J. M ater. Sci. M ater. $M$ ed. (2007) (Epub ahead of print).

22. Duan $Y$, Wang Z, Yan W, Wang S, Zhang $S$, Jia J: Preparation of collagen-coated electrospun nanofibers by remote plasma treatment and their biological properties. J. Biomater. Sci. Polym. Ed. 18, 1153-1164 (2007).

23. Li W, Guo Y, Wang $\mathrm{H}$ et al.: Electrospun nanofibers immobilized with collagen for neural stem cells culture. J. M ater. Sci. $M$ ater. M ed. (2007) (Epub ahead of print).

24. Christman KL, Enriquez-Riosa VD, $M$ aynard H D : N anopatterning proteins and peptides. Soft M atter 2, 928-939 (2006).

25. Comisar WA, Kazmers N H , M ooney DJ, Linderman JJ: Engineering RGD nanopatterned hydrogels to control preosteoblast behavior: a combined computational and experimental approach. Biomaterials 28, 4409-4417 (2007). 